\title{
The semeiology of arthritis: discriminating between patients on the basis of their symptoms
}

\author{
P S Helliwell
}

\begin{abstract}
Objectives-To examine the intended meaning of words used by patients to describe arthritic symptoms, and to distinguish between different patient groups on the basis of these words.

Methods-A Joint Symptom Questionnaire, developed to resemble the McGill Pain Questionnaire, was given to health professionals $(n=50)$ and patients with rheumatoid arthritis $(R A)(n=100)$, fibromyalgia (FM) ( $=50)$, ankylosing spondylitis (AS) $(n=50)$, and osteoarthritis (OA) $(n=50)$. Respondents were invited to define each word by selecting an appropriate heading. Comparison of patient groups was based on the selection of words they chose to describe their joint symptoms.

Results-Between health professionals and patients there were no semantic differences in the words given. Patients with FM chose more words to describe their symptoms than the other patient groups (RA median nine words; AS nine words; OA 10 words; FM 12 words). Using receiver operating characteristic curves, a clear distinction between patients with RA, FM, and AS was found, but patients with RA were not readily separated from patients with $O A$.

Conclusions-There appears to be no semeiological confusion between health professionals and patients regarding arthritic symptoms. However, the spectrum of words chosen by patients to describe the feelings in their joints permits a separation between patients with RA, AS, and FM. Using this questionnaire, patients with $R A$ and $O A$ are symptomatically similar.
\end{abstract}

(Ann Rheum Dis 1995; 54: 924-926)

Although stiffness has headed the list of diagnostic criteria for rheumatoid arthritis for more than 30 years $^{12}$ and has remained a major outcome variable in many studies of the efficacy of antirheumatic drugs and physical therapy, this pre-eminence has been challenged. Abrahamson ${ }^{3}$ was of the opinion that the symptom of stiffness was difficult to elicit because patients variously described stiffness as numbness, weakness, aching, and other discomforts. More recently, a cohort of 97 patients, when prompted, provided 13 other adjectives to describe their joint symptoms, ${ }^{4}$ suggesting that physicians may artificially restrict patients to using certain descriptors (pain and stiffness).

According to the Shorter Oxford English Dictionary ${ }^{5}$ semeiology is the branch of medical science that is concerned with symptoms; semantics is the branch of linguistics that deals with the study of meaning. Burge ${ }^{6}$ has suggested that people may wrongly describe sensations attributable to arthritis based on communal conventions governing figures of speech. This would suggest that patients and health professionals may have different understandings of the words used to describe arthritic symptoms, and this confusion may underlie the discrepancy between subjective and objective stiffness in rheumatoid arthritis. ${ }^{7}$

A new questionnaire similar to the McGill Pain Questionnaire (MPQ) ${ }^{8}$ was developed, and administered to patients and health professionals to obtain a semantic comparison of symptoms. Examination of descriptors chosen by patients with different arthritic diseases (rheumatoid arthritis (RA), fibromyalgia (FM), ankylosing spondylitis (AS) and osteoarthritis (OA)) then enabled a symptom profile of these disorders to be compared.

\section{Methods}

DEVELOPMENT OF QUESTIONNAIRE

A list of 55 words, descriptors used to describe sensations arising from joints, was compiled as follows: 29 words from descriptions given by patients who had RA according to revised American Rheumatism Association (ARA) criteria, ${ }^{2}$ and were asked to list as many words as possible that best described how their joints felt; an additional five words were taken from the literature: ${ }^{4}$ aches, hurts, tense, inflexible, and painful; 19 synonyms to the above descriptors, derived from a thesaurus ${ }^{9}$ were included; two other words were added from the MPQ (throbbing and pulling), together with 10 other words already identified as common to the MPQ (cramping, cold, numb, tight, taut, squeezing, heavy, aching, hurting, and sore).

This total list of 55 words was then presented to a group of health professionals. Subjects were invited to categorise each of the words under any of eight headings: weakness, friction, limited range of movement, pain, swelling, resistance to movement, lack of feeling, and lack of movement. Fifty replies 
Appropriate category headings for the descriptors according to 50 health professionals

\begin{tabular}{ll}
\hline Category & Descriptors (\% agreement) \\
\hline Weakness & Lethargic (78), weak (98), heavy (40) \\
Friction & Creaking (92), grating (100), grinding (98) \\
Limited range of movement & Limited (90), restricted (78) \\
Pain & Aches (98), hurts (98), painful (100), sore (96) \\
Swelling & Puffy (100), tight (52), tense (42) \\
Resistance to movement & Stiff (64), stubborn (80) \\
No feeling & Cold (82), numb (96), wooden (62) \\
Lack of movement & Fixed (82), inflexible (56), jammed (72), locked (78), rigid (82), \\
& set (82), solid (84), stuck (76) \\
\hline
\end{tabular}

were received comprising medical staff (16), physiotherapists (11), bioengineers (eight), nursing staff (seven), occupational therapists (four), secretarial staff (two), a pharmacist (one), and a social worker (one). As in the MPQ , 60\% agreement between respondents was sought as a cut off for agreed representation of a particular word in a particular class. Using this criterion, 24 descriptors were categorised; in addition, four words were added as they formed part of the original patient list but achieved concordance rates of less than $60 \%$. In the final list of 28 words, 19 originated from patients, five from the rheumatological literature, and four from the thesaurus (table).

\section{PATIENTS}

The majority of patients were attending outpatient clinics: all had English as a first language. Patients with RA all satisfied ARA diagnostic criteria. $^{2}$ They comprised 100 patients, mean age 58.6 years, 67 women, 33 men. Patients with AS (50 patients, mean age 40.6 years, six women, 44 men) were diagnosed on the basis a history of chronic spinal pain, reduced range of motion, and radiological sacroiliitis. ${ }^{10}$ Patients with FM (50 patients, mean age 46.4 years, 41 women, nine men) were diagnosed on the basis of widespread chronic, non-articular pain with normal radiology, normal serology, and normal plasma viscosity; patients had at least four tender

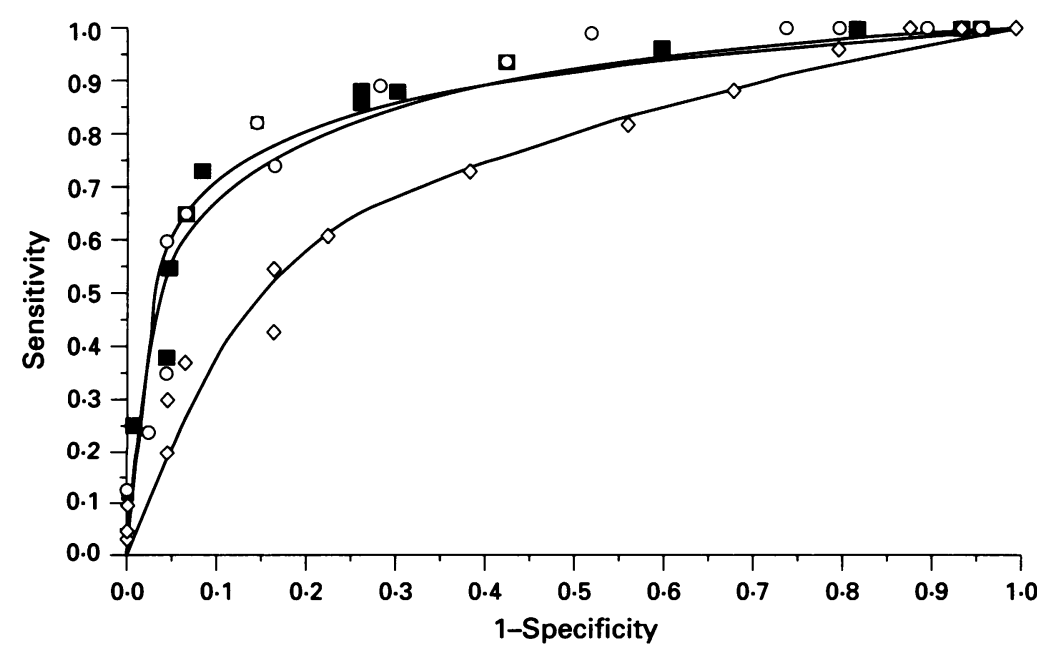

Receiver operating characteristic curves for rheumatoid arthritis (RA) compared with fibromyalgia (FM) (D), ankylosing spondylitis $(A S)(O)$, and osteoarthritis $(O A)(\diamond)$. For each comparison (curve) the more the curve is convex upwards to the left, the better the discriminatory power of the questionnaire; a comparison with no discriminatory power would show a straight line from the bottom left to the top right corner of the graph. points, including the upper border of the trapezius muscle. The patients with OA (50 patients, mean age 66.3 years, 40 women and 10 men) were diagnosed on clinical and radiological criteria: predominantly, they had OA of a major joint such as the knee.

Patients were handed the questionnaire and were asked to work through the list and arrange each of the words under one of the eight category headings given. In addition, patients were asked to underline any word which they felt described their joint symptoms. The patients took the questionnaire away with them, completed it elsewhere, and returned it in a stamped addressed envelope provided.

\section{ANALYSES OF FINDINGS}

The effect of age and gender on descriptor choice may confound any attempt to discriminate between disease groups, therefore the percentage of respondents selecting each descriptor was compared between the sexes and between two age groups using the median age as cut off.

To examine the ability of the descriptors to discriminate between disease groups, logistic regression was performed using the disease group as the dependent variable, and the descriptors as independent variables. Separate logistic regressions were carried out for rheumatoid arthritis against each of the other disease groups. For each patient the following statistic was then calculated:

$$
L=\ln (\mathrm{p} / 1-\mathrm{p})=b_{0}+b_{1} x_{1}+b_{2} x_{2}+\ldots b_{\mathrm{i}} x_{\mathrm{i}}
$$

where $\mathrm{p}=$ probability of having disease (range $0-1) ; b_{0}=$ constant; $b_{\mathrm{i}}=$ coefficient of $\mathrm{ith}$ descriptor; $x_{\mathrm{i}}=$ response to ith descriptor $(0$ or 1$)$.

Receiver operating characteristic (ROC) curves were then constructed using different values of $L$ as cut off between $\mathrm{RA}$ and the comparator disease (figure). The area under the ROC curve was calculated according to the method of Hanley and McNeil. ${ }^{11}$

\section{Results}

PATIENT CATEGORISATION OF DESCRIPTORS Despite all questionnaires being returned, completion of the questionnaire, particularly with regard to sorting each of the descriptors under a category heading, was variable. Words commonly not sorted were: stubborn, stuck, solid, and wooden. Overall, $61 \%$ of patients categorised all the descriptors.

Analysis was restricted to the 24 descriptors achieving greater than $60 \%$ concordance by health professionals. As for the health professionals, each descriptor was matched against the category heading with the greatest concordance. For all of the descriptors there was complete agreement between patients and health professionals. For each of the patient groups and the health professionals, the category most appropriately defining the descriptor 'stiff' was 'resistance to movement'. Alternative categories chosen included 'limited range of movement' and 'lack of movement'. 
USE OF DESCRIPTORS BETWEEN DIFFERENT DISEASES

Descriptors were underlined by 244 of 250 patients. The median number of words underlined by patients was as follows: RA nine words, OA 10 words, AS nine words, FM 12 words (Kruskall-Wallis, all groups, $p=0.26$; Mann-Whitney RA and AS $v$ FM, $\mathrm{p}=0.05$ ). Patients with FM commonly chose all the pain descriptors plus other affective descriptors such as 'grinding', 'heavy', and 'lethargic'.

Only minor differences in descriptor choice were found between the sexes and between two age groups.

There was good discrimination between RA and FM, and between RA and AS, but poor discrimination between RA and OA: calculated areas under the ROC curve were 0.93 (95\% confidence intervals $0.88-0.97$ ) for RA $v$ FM, $0.73(0.64-0.82)$ for RA $v \mathrm{OA}$, and 0.89 $(0.83-0.95)$ for RA $v$ AS.

\section{Discussion}

This questionnaire met the aims of the study adequately from both a semantic and a semeiological point of view. Although the word 'stiffness' retains a pre-emptive place in the diagnostic criteria for RA, doubts have been raised about patients' understanding of this word. ${ }^{7}$ This study has shown that patients feel able to distinguish between pain and stiffness, the latter indicating increased resistance to movement, and limited range or lack of movement. Recent work has clarified the essentially biomechanical problem of measuring increased resistance to movement, and has vindicated the patients' description of sensations arising from the joint. ${ }^{12}$

Although the questionnaire was designed to resemble the $M P Q$, further development would be needed to provide the questionnaire with a structure similar to that seen for the evaluation of pain. Modification of the questionnaire to allow patients to express the intensity and duration of descriptors would give a structure similar to that of the MPQ and may improve its discriminatory power.

In some ways it is surprising that by the use of joint descriptors alone it was possible to discriminate between patients with rheumatoid arthritis, fibromyalgia and ankylosing spondylitis. It is possible that our patients become conditioned to use certain joint descriptors after repeated exposure to physicians who use distinctive prompts, possibly a different set according to each disease. However, Leavitt et $a l,{ }^{13}$ comparing patients with FM and those with RA using a modified MPQ, found that patients with FM used words with greater spatial diffusion and words less localised to the joints and, furthermore, used evaluative words more commonly. In addition, patients with FM chose significantly more pain descriptors than those with RA-a finding similar to those of this study. It does seem therefore that, in a forced choice situation, patients with different diseases will select an overlapping but distinctly separate spectrum of descriptors to describe their rheumatic symptoms.

In contrast with the discrimination achieved between RA, FM, and AS, OA and RA were difficult to separate using this questionnaire. Charter et $a l^{14}$ also encountered this difficulty using the MPQ, with which a similar word choice for both patient groups was found. Hazes $e t a l^{15}$ found no difference between OA and RA in terms of duration or severity of early morning stiffness. Published studies would, therefore, seem to show that patients with RA and those with OA are difficult to separate on a purely symptomatic basis. Perhaps the addition of the dimension of intensity would provide better separation between these groups.

In summary, it is important to acknowledge that this is not a diagnostic test for different rheumatic diseases, and that further development of the quetionnaire is required before it can be used in a manner similar to the MPQ. The use of this questionnaire in different disease groups suggested that a clear distinction can be made between rheumatoid arthritis, fibromyalgia and ankylosing spondylitis on symptoms alone. If further information on the site and intensity of the symptoms could be included, this might further aid distinction between the patient groups.

I wish to thank the staff of the Rheumatology and Rehabilitation Research Unit for their help in development of the questionnaire, and Mrs $M$ Turner for preparing the manuscript.

1 Ropes M W, Bennett F A, Cobb S, et al. 1958 revision of diagnostic criteria for rheumatoid arthritis. Arthritis Rheum 1959; 2: 16-20

2 Arnett F C, Edworthy S M, Bloch D A, et al. The American Rheumatism Association 1987 revised criteria for the classification of rheumatoid arthritis. Arthritis Rheum 1988; 31: 315-24.

3 Abramson J H. On the diagnostic criteria of active rheumatoid arthritis. $f$ Chron Dis 1967; 20: 275-90

4 Rhind V M, Unsworth A, Haslock I. Assessment of stiffness in theumatology - the use of rating scales. $B r \mathcal{F}$ Rheumatol 1987; 26: 126-30.

5 Shorter Oxford English Dictionary. Oxford: Oxford University Press, 1973.

6 Burge T. Fundamentalism and the mental. Midwest Studies in Philosophy, Vol 4, Studies in metaphysics. Minnesota: University of Minnesota Press, 1979; 73-121.

7 Helliwell P S, Howe A, Wright V. Lack of objective evidence of stiffness in rheumatoid arthritis. Ann Rheum Dis 1988; 47: 754-8

8 Melzack R. The McGill Pain Questionnaire: major properties and scoring methods. Pain 1975; 1: 277-99.

9 Roget's Thesaurus. London: Longman Group, 1962.

10 Bennett P H, Burch T A. New York symposium on population studies in the rheumatic diseases-new diagnostic criteria. Bull Rheum Dis 1967; 17: 453-8.

11 Hanley J A, McNeil B J. The meaning and use of the area under a receiver operating characteristic (ROC) curve.
Radiology 1982; 143: 29-36.

12 Helliwell P S, Smeathers J E, Wright V. The contribution of different tissues to stiffness of the wrist joint. Proc Inst of different tissues to stiffness of the

13 Leavitt F, Katz R S, Golden H E, et al. Comparison of pain properties in fibromyalgia patients and rheumatoid arthritis patients. Arthritis Rheum 1986; 29: 775-81.

14 Charter R A, Nehemkis A M, Keenan M A, Person D, Prete $\mathrm{P}$. The nature of arthritis pain. Br $\mathcal{f}$ Rheumatol 1985 ; 24: $53-60$.

15 Hazes J M W, Hayton R, Silman A J. A re-evaluation of the symptom of morning stiffness. $\mathcal{F}$ Rheumatol 1993; 20: $1138-42$. 